\title{
Stationary Black Holes and Light Rings
}

\author{
Pedro V. P. Cunha $\odot^{1}$ and Carlos A. R. Herdeiro $\odot^{2}$ \\ ${ }^{1}$ Max Planck Institute for Gravitational Physics-Albert Einstein Institute, Am Mühlenberg 1, Potsdam 14476, Germany \\ ${ }^{2}$ Departamento de Matemática da Universidade de Aveiro and CIDMA, Campus de Santiago, 3810-183 Aveiro, Portugal
}

(Received 19 March 2020; accepted 15 April 2020; published 8 May 2020)

\begin{abstract}
The ringdown and shadow of the astrophysically significant Kerr black hole (BH) are both intimately connected to a special set of bound null orbits known as light rings (LRs). Does it hold that a generic equilibrium BH must possess such orbits? In this Letter we prove the following theorem. A stationary, axisymmetric, asymptotically flat black hole spacetime in $1+3$ dimensions, with a nonextremal, topologically spherical, Killing horizon admits, at least, one standard LR outside the horizon for each rotation sense. The proof relies on a topological argument and assumes $C^{2}$ smoothness and circularity, but makes no use of the field equations. The argument is also adapted to recover a previous theorem establishing that a horizonless ultracompact object must admit an even number of nondegenerate LRs, one of which is stable.
\end{abstract}

DOI: 10.1103/PhysRevLett.124.181101

Introduction.-The second decade of the 21 st century will be celebrated as the dawn of precision strong gravity. New observational data is testing, in particular, the true nature of astrophysical black holes (BHs). Both gravitational wave observations $[1,2]$, notably through the ringdown phase, and electromagnetic observations, in particular through the shadow imaging [3-5], are expected to provide hitherto inaccessible information on the $\mathrm{BH}$ spacetime geometry.

The ringdown and shadow observables are both intimately connected to a special set of bound null orbits for test particles [6,7]. When planar, these orbits are known as light rings (LRs). They are an extreme form of light deflection, such that the path of light closes over itself. In the general nonplanar case these light paths are dubbed fundamental photon orbits (FPOs) [8]. For a spherical BH, such as the Schwarzschild solution, all FPOs are LRs. This is not so for axisymmetric, but nonspherical, BHs. In the special case of the Kerr spacetime, the FPOs are known as spherical photon orbits [9], all of which are unstable (in the radial direction) outside the horizon and reduce, in two appropriate limits, to LRs. The latter correspond to equatorial photon orbits which are corotating or counterrotating with the Kerr horizon.

The close connection between LRs and the aforementioned key observables raises the following question: Does an equilibrium BH spacetime always possess LRs? This is the case for the paradigmatic electro-vacuum BHs of General Relativity (GR), but can one safely extrapolate to BHs with generic matter contents or modified gravity?

In this Letter we shall provide a generic and robust answer to these questions using a topological argument. Concretely, under reasonable assumptions, we shall establish the following theorem: A stationary, axisymmetric, asymptotically flat, $1+3$ dimensional $\mathrm{BH}$ spacetime,
$(\mathcal{M}, g)_{\mathrm{BH}}$, with a nonextremal, topologically spherical Killing horizon, $\mathcal{H}$, admits at least one standard LR outside the horizon for each rotation sense.

The spacetime.-We assume an equilibrium $\mathrm{BH}$ spacetime under the conditions of the last paragraph. No assumption is made on the field equations $(\mathcal{M}, g)_{\mathrm{BH}}$ solves. This spacetime possesses two Killing vectors $\{\xi, \eta\}$, associated, respectively, to stationarity and axisymmetry. Asymptotic flatness implies $\{\xi, \eta\}$ must commute [10]. Then, coordinates $(t, \varphi)$ adapted to the Killing vectors $\xi=\partial_{t}, \eta=\partial_{\varphi}$ can be chosen. In addition, we assume that the metric is at least $C^{2}$-smooth on and outside $\mathcal{H}$, and circular. The latter, together with asymptotic flatness, implies the spacetime admits a 2-space orthogonal to $\left\{\partial_{t}, \partial_{\varphi}\right\}$ 一see, e.g., Theorem 7.11 in Ref. [11]. This means the metric $g$ possesses a discrete symmetry $(t, \varphi) \rightarrow$ $(-t,-\varphi)[12]$.

In the orthogonal 2-space one can introduce sphericallike coordinates $(r, \theta)$. The sections of $\mathcal{H}$ are assumed to be topologically spherical. A gauge choice guarantees the horizon is located at a constant (positive) radial coordinate $r=r_{H}$. The polar coordinate $\theta$ is chosen to be always orthogonal to $r$. In such a gauge, $g_{r \theta}=0, g_{r r}>0$ and $g_{\theta \theta}>$ 0 outside $\mathcal{H}$. One can further require that $(r, \theta)$ reduce to standard spherical coordinates in the asymptotically flat limit $r \rightarrow \infty$. The coordinates' range is then, outside the horizon, $r \in\left[r_{H}, \infty[, \theta \in[0, \pi]\right.$ with $\theta=\{0, \pi\}$ at the rotation axis, $\varphi \in[0,2 \pi[$ and $t \in]-\infty,+\infty[$. Outside $\mathcal{H}$, causality requires $g_{\varphi \varphi} \geq 0$. The metric, which has a Lorenzian signature $(-,+,+,+)$, thus reads $d s^{2}=g_{t t} d t^{2}+$ $2 g_{t \varphi} d t d \varphi+g_{\varphi \varphi} d \varphi^{2}+g_{r r} d r^{2}+g_{\theta \theta} d \theta^{2}$.

The Killing horizon.-The existence of $\mathcal{H}$ means there is a Killing vector field, $\chi=\partial_{t}+\omega_{H} \partial_{\varphi},\left(\omega_{H}=\right.$ const $)$ that is null on $\mathcal{H},\left.\left(\chi^{\mu} \chi_{\mu}\right)\right|_{\mathcal{H}}=0$. Then, $\chi$ is the horizon null 
generator. For stationary BHs, one can further introduce a (positive) constant quantity on $\mathcal{H}$, the surface gravity $\kappa$, defined via the following relation computed at the horizon $\left.\left[\nabla_{\mu}\left(\chi^{2}\right)=-2 \kappa \chi_{\mu}\right]\right|_{\mathcal{H}}$. Taking $\mu \in\{t, \varphi\}$, one obtains $0=\left.\left(g_{\mu t}+g_{\mu \varphi} \omega_{H}\right)\right|_{\mathcal{H}}$. This implies that $\omega_{H}=$ $-\left.\left(g_{t \varphi} / g_{\varphi \varphi}\right)\right|_{\mathcal{H}}$, for the horizon angular velocity $\omega_{H}$, and $\left.D\right|_{\mathcal{H}}=0$, where we have defined $D \equiv\left(g_{t \varphi}^{2}-g_{t t} g_{\varphi \varphi}\right)$. Thus, $D$ vanishes on $\mathcal{H}$; in fact, it is positive outside the horizon and away from the axis [13].

LRs and a topological charge.-For diagnosing the occurrence of LRs in $(\mathcal{M}, g)_{\mathrm{BH}}$, one must consider the null geodesic flow. Following Ref. [14], LRs are identified by considering the effective potentials on the orthogonal 2-space, $H_{ \pm}$

$$
H_{ \pm}(r, \theta) \equiv \frac{-g_{t \varphi} \pm \sqrt{D}}{g_{\varphi \varphi}}
$$

LRs are critical points of $H_{ \pm}$[15]; a LR obeys either $\partial_{\mu} H_{+}=0$ or $\partial_{\mu} H_{-}=0$ or both simultaneously (e.g., for static spacetimes) [16]. The \pm sign is typically associated with the two possible rotation senses (see Supplemental Material [17], Sec. I).

We can associate a topological charge to LRs. First, introduce a field $\mathbf{v}=\left(v_{r}, v_{\theta}\right)$ as a normalized gradient of $H_{ \pm}$:

$$
v_{r} \equiv \frac{\partial_{r} H_{ \pm}}{\sqrt{g_{r r}}}, \quad v_{\theta} \equiv \frac{\partial_{\theta} H_{ \pm}}{\sqrt{g_{\theta \theta}}} .
$$

If follows that $\partial^{\mu} H_{ \pm} \partial_{\mu} H_{ \pm}=v_{r}^{2}+v_{\theta}^{2} \equiv v^{2}$. Hence, in terms of $\mathbf{v}$, a LR occurs if and only if $\mathbf{v}=0 \Leftrightarrow v=0$.

Second, define an angle $\Omega$ such that $v_{r}=v \cos \Omega$, $v_{\theta}=v \sin \Omega$. Then, $\Omega$ together with the "norm" $v$, parameterizes the auxiliary 2 -space spanned by $\mathbf{v}$, denoted $\mathcal{V}$.

Third, in the physical orthogonal 2-space $(r, \theta)$, consider a simple closed curve $C$, that is piecewise smooth and positive oriented. Since $C$ is closed, the angle $\Omega$ after a full revolution must be the same, modulo $2 \pi$. Hence,

$$
\oint_{C} d \Omega=2 \pi w, \quad w \in \mathbb{Z} .
$$

In the physical $(r, \theta)$ space $w$ counts the winding number of $\mathbf{v}$ as $C$ is circulated in the positive sense. When $C$ encloses a single (nondegenerate $[14,18]$ ) LR, the integer $w$ is the topological charge of the LR. Indeed, the curve $C$, in the physical $(r, \theta)$ space, defines a curve $\tilde{C}$ in $\mathcal{V}$, via Eq. (2). In $\mathcal{V}, w$ is the winding number of $\tilde{C}$ around the origin $(v=0)$, which corresponds to a LR. Thus, in $\mathcal{V}, w$ constitutes a well-defined topological quantity [19]: deforming $\tilde{C}$ without crossing the origin does not change $w$. Consequently, in the physical $(r, \theta)$ space, deforming $C$ without crossing a LR does not change $w$.

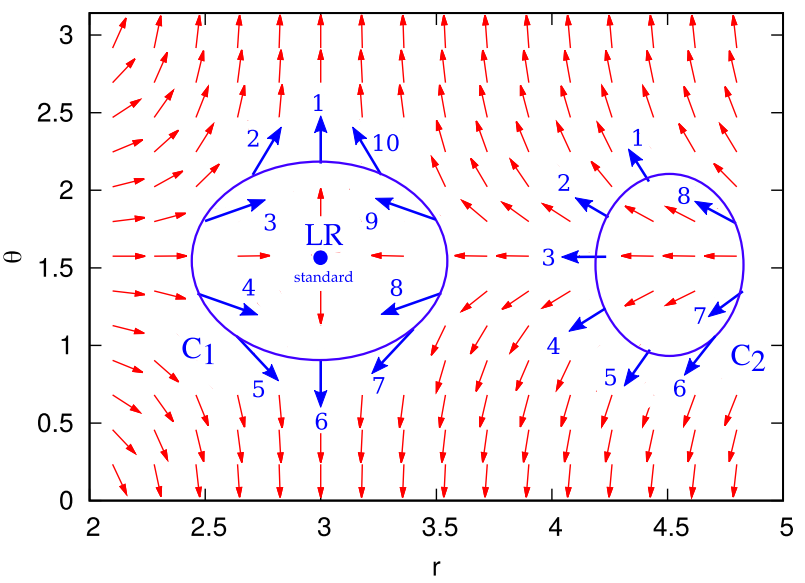

FIG. 1. The red arrows represent $\mathbf{v}$ (normalized to unity), defined from Eq. (2) with $H_{+}=\sqrt{1-2 / r} /(r \sin \theta)$, on a portion of the $(r, \theta)$ plane for the Schwarzschild $\mathrm{BH}$ with unit mass, in standard coordinates. The LR sits at $r=3, \theta=\pi / 2$. Circulating the contour $C_{1}$ (or any contour that encloses the LR) anticlockwise, $\mathbf{v}$ winds once clockwise (follow the blue arrows $1 \rightarrow 10$ ). Thus $w=-1$. By contrast, circulating the contour $C_{2}$ (or any contour that does not enclose the LR) anticlockwise, $\mathbf{v}$ has no winding. Thus $w=0$. Observe two important properties that will be general. (1) $\mathbf{v}$ becomes vertical at $\theta=0(\theta=\pi)$ and downwards (upwards) directed; (2) $v_{r}$ is positive (negative) as the horizon (asymptotic infinity) is approached. The signs are reversed for $H_{-}$.

Figure 1 exhibits $\mathbf{v}$ for a Schwarzschild BH. It illustrates that $w=-1(w=0)$ for any contour that encloses (does not enclose) the Schwarzschild LR. In general, if $C$ encloses a single saddle point (maximum or minimum) of the potential $H_{ \pm}(r, \theta)$, then $w=-1(w=+1)$. A LR with $w=-1(w=+1)$ is dubbed standard (exotic). LRs in Schwarzschild and Kerr are standard. Furthermore, for any $C$, the total $w$ is the sum of the individual LR charges within $C$. In particular, if there are no LRs within $C$, then $w=0$.

Our task is to show that the total LR topological charge in the region outside a $\mathrm{BH}$ (under the assumptions stated above) is $w=-1$, regardless of choosing $H_{+}$or $H_{-}$. This implies that at least one standard LR must exist within that region, for each rotation sense of the $\mathrm{BH}$, and establishes the theorem. To achieve this we must select an appropriate contour.

The contour.-For our generic $(\mathcal{M}, g)_{\mathrm{BH}}$, we define a contour $C$ that encompasses a subregion $\mathcal{I}$ of the orthogonal 2-space exterior to the horizon. Then, taking appropriate limits, $\mathcal{I}$ becomes the full exterior region.

The region $\mathcal{I}$ is shown in Fig. 2 and it is defined as $r_{0} \leq r \leq R$ and $\delta \leq \theta \leq \pi-\delta$. The constants $\left\{r_{0}, R, \delta\right\}$ are such that $r_{H}<r_{0} \ll R$ and $0<\delta \ll 1$.

$\mathcal{I}$ is the region enclosed by the curve $C$ (see Fig. 2), which is defined as the union of four line segments: $\{r=R, \delta \leq \theta \leq \pi-\delta\} \cup\left\{\theta=\pi-\delta, r_{0} \leq r \leq R\right\} \cup\left\{r=r_{0}\right.$, $\delta \leq \theta \leq \pi-\delta\} \cup\left\{\theta=\delta, r_{0} \leq r \leq R\right\}$. 


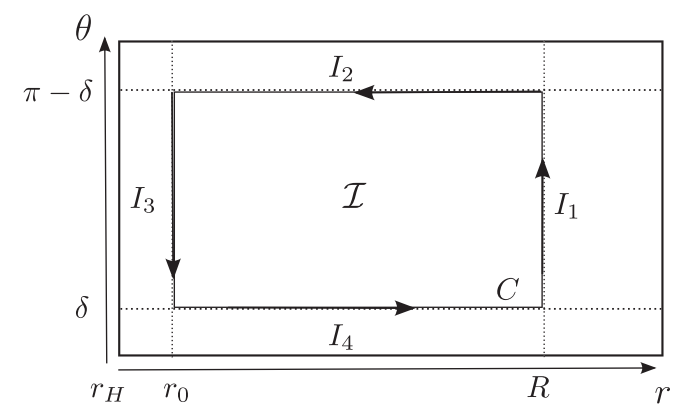

FIG. 2. Representation of the contour $C$ (which encloses $\mathcal{I}$ ) on the $(r, \theta)$ plane. The curve $C$ has positive orientation and it is composed by four line segments.

The topological charge of $\mathcal{I}$ is computed from Eq. (3), decomposed as $2 \pi w_{\mathcal{I}}=I_{1}+I_{3}+I_{2}+I_{4}$, where

$I_{1}=\left[\int_{\delta}^{\pi-\delta} \frac{d \Omega}{d \theta} d \theta\right]_{r=R}, \quad I_{2}=\left[\int_{R}^{r_{0}} \frac{d \Omega}{d r} d r\right]_{\theta=\pi-\delta}$,

$I_{3}=\left[\int_{\pi-\delta}^{\delta} \frac{d \Omega}{d \theta} d \theta\right]_{r=r_{0}}, \quad I_{4}=\left[\int_{r_{0}}^{R} \frac{d \Omega}{d r} d r\right]_{\theta=\delta}$.

To obtain the total topological charge of the exterior region, we take first $\delta \rightarrow 0$ (axis limit), and only then $r_{0} \rightarrow$ $r_{H}$ (horizon limit) and $R \rightarrow+\infty$ (asymptotic limit):

$$
w=\lim _{R \rightarrow+\infty} \lim _{r_{0} \rightarrow r_{H}}\left(\lim _{\delta \rightarrow 0} w_{\mathcal{I}}\right)
$$

These limits must be taken with care, as we now discuss.

Axis limit.-The axis is the set of points for which $g_{\varphi \varphi}=\eta \cdot \eta=0=\eta \cdot \xi=g_{t \varphi}$. To approach the axis, introduce a local coordinate $\rho$, defined as $\rho \equiv \sqrt{g_{\varphi \varphi}}$ (recall $g_{\varphi \varphi}>0$ outside $\mathcal{H}$ ). Clearly, $d \rho / d \theta$ is positive (negative) as $\theta \rightarrow 0(\theta \rightarrow \pi)$. Then, consider a small $\rho$ expansion close to the axis:

$$
\begin{gathered}
g_{\varphi \varphi}=\rho^{2}, \quad g_{t \varphi} \simeq b_{o} \rho^{n}+\mathcal{O}\left(\rho^{n+1}\right), \\
g_{t t} \simeq g_{t t}^{0}+\mathcal{O}(\rho), \quad g_{\rho \rho} \simeq g_{\rho \rho}^{0}+\mathcal{O}(\rho),
\end{gathered}
$$

where $n \in \mathbb{N}$ and some constants were introduced. By assuming $C^{2}$ smoothness and regularity (e.g., a nondiverging Ricci scalar) close to the axis $g_{\varphi \varphi}$ cannot go to zero faster than $g_{t \varphi}$ in the axis limit (see Supplemental Material [17], Sec. II and Ref. [20]). Then $2 \leq n$ and $\rho^{2 n} \ll \rho^{2}$. It follows from the definition of $D$ that $\sqrt{D} \simeq \rho \sqrt{-g_{t t}^{0}}$. Hence, from Eq. (1):

$$
H_{ \pm} \simeq \pm \frac{\sqrt{-g_{t t}^{0}}}{\rho}
$$

One can now estimate $\mathbf{v}$ from Eq. (2). In particular, using $g_{\rho \rho} d \rho^{2} \simeq g_{\theta \theta} d \theta^{2}$ at zeroth order in $\rho$ :
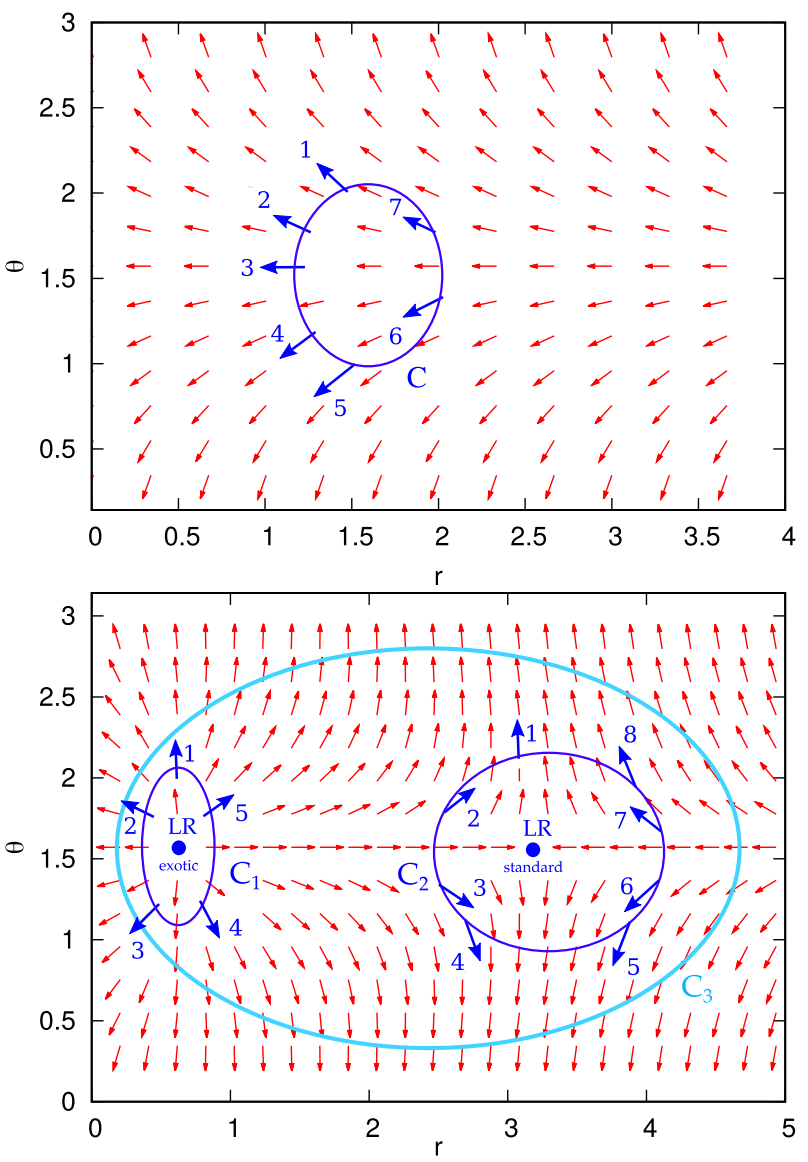

FIG. 3. Top: The red arrows represent $\mathbf{v}$, defined from Eq. (2) with $H_{+}=1 /(r \sin \theta)$, on a portion of the $(r, \theta)$ plane for flat spacetime, in standard coordinates. There are no LRs. Observe the key difference with respect to Fig. 1 . Here, $v_{r}$ is negative (positive for $H_{-}$) as the left boundary of the domain is approached, which is now a regular origin at $r=0$, rather than a horizon. Any contour $C$ will have $w=0$. Bottom: $\mathbf{v}$ (defined from $H_{+}$) for a horizonless ultracompact object (rotating boson star). There are two LRs with opposite topological charge. By circulating the contour $C_{1}\left(C_{2}\right)$ anticlockwise, $\mathbf{v}$ winds once in the positive (negative) sense (follow the numbered blue arrows). By contrast, after a full circulation along $C_{3}$, which encompasses both LRs, $\mathbf{v}$ winds up zero times $(w=0)$.

$$
v_{\theta} \simeq \operatorname{sign}\left(\frac{d \rho}{d \theta}\right) \frac{\partial_{\rho} H_{ \pm}}{\sqrt{g_{\rho \rho}}} \sim \mp \operatorname{sign}\left(\frac{d \rho}{d \theta}\right) \frac{1}{\rho^{2}} .
$$

Since $v_{\theta} \sim \rho^{-2}$ and $v_{r} \sim \rho^{-1}$, then $v_{\theta}^{2} \gg v_{r}^{2}$, and so $v \simeq\left|v_{\theta}\right|$. Hence as $\rho \rightarrow 0$ one obtains $v_{\theta} / v \rightarrow \mp \operatorname{sign}(d \rho / d \theta)$. Consequently,

$$
\Omega=\left.\arcsin \left(\frac{v_{\theta}}{v}\right)\right|_{0, \pi} \rightarrow \begin{cases} \pm \pi / 2 & \text { for } \theta \rightarrow \pi \\ \mp \pi / 2 & \text { for } \theta \rightarrow 0\end{cases}
$$

The axis limit is $\lim _{\delta \rightarrow 0} C$, which implies $\rho \rightarrow 0$ along the integration paths of $\left\{I_{2}, I_{4}\right\}$. Thus, the bottom line is that $\Omega$ becomes constant along the integration path. 
Consequently, the contribution of $\left\{I_{2}, I_{4}\right\}$ to $w$ vanishes as $\delta \rightarrow 0$.

This result can be interpreted as follows. In a generic $\mathrm{BH}$ spacetime, the arrows analogous to those in Fig. 1 become vertical along $\left\{I_{2}, I_{4}\right\}$ as $\delta \rightarrow 0$, directed upwards (downwards) at $\theta=\pi$ and downwards (upwards) at $\theta=0$, for $H_{+}\left(H_{-}\right)$. Hence, the integration along these paths does not contribute to the winding of $\mathbf{v}$, as $C$ is circulated.

Horizon limit.-To address the horizon limit $\left(r_{0} \rightarrow r_{H}\right)$ we observe that, as discussed in Ref. [21], the metric near the Killing horizon of a generic stationary and axially symmetric BH is fairly constrained if we require regularity (e.g., finite Ricci scalar at horizon). If the BH is not extremal $(\kappa \neq 0)$, we can set a local radial coordinate $x$ such that $g_{x x}=1$ and $\left.x\right|_{\mathcal{H}}=0$ at the horizon. We also define $N=\sqrt{D / g_{\varphi \varphi}}$ and $\omega=-g_{t \varphi} / g_{\varphi \varphi}$, which yields $H_{ \pm}=$ $\omega \pm N / \sqrt{g_{\varphi \varphi}}$. Then, near the horizon [21]

$\omega \simeq \omega_{H}+\mathcal{O}\left(x^{2}\right), \quad N \simeq \kappa x+\mathcal{O}\left(x^{3}\right), \quad g_{\varphi \varphi} \simeq g_{\varphi \varphi}^{H}+\mathcal{O}\left(x^{2}\right)$.

This leads to

$$
\partial_{x} H_{ \pm} \simeq \pm \frac{\kappa}{\sqrt{g_{\varphi \varphi}^{H}}}+\mathcal{O}(x) .
$$

Since $\left(1 / \sqrt{g_{x x}}\right)(\partial / \partial x)=\left(1 / \sqrt{g_{r r}}\right)(\partial / \partial r)$, then near the horizon $(x \simeq 0)$ :

$$
v_{r}=\frac{\partial_{r} H_{ \pm}}{\sqrt{g_{r r}}} \simeq \pm \frac{\kappa}{\sqrt{g_{\varphi \varphi}^{H}}} .
$$

Thus, we have the following horizon limit:

$$
\left.\operatorname{sign}\left(v_{r}\right)\right|_{\mathcal{H}}= \pm 1 \text {. }
$$

This is sufficient for our purpose. It means that $\mathbf{v}$ has a positive (negative) radial component along $I_{3}$ for $H_{+}\left(H_{-}\right)$, in the horizon limit. By continuity, along $I_{3} \mathbf{v}$ interpolates between an upwards (downwards) directed $\mathbf{v}$ at the intersection with $I_{2}$ - see Fig. 1 -and a downwards (upwards) directed $\mathbf{v}$ at the intersection with $I_{4}$, for $H_{+}\left(H_{-}\right)$. Its positive (negative) radial component along $I_{3}$, means $\mathbf{v}$ winds in the negative, i.e., clockwise, direction along $I_{3}$, producing half of a full winding. Thus

$$
\Omega_{\theta=0}^{\mathcal{H}}-\Omega_{\theta=\pi}^{\mathcal{H}}=-\pi
$$

Asymptotic limit.-Finally consider the limit $R \rightarrow \infty$ (integration path of $I_{1}$ ). One reaches flat spacetime in standard spherical coordinates, yielding

$$
\left.v_{r} \simeq \mp \frac{1}{r^{2} \sin \theta} \Rightarrow \operatorname{sign}\left(v_{r}\right)\right|_{\infty}=\mp 1 .
$$

Again, this information suffices: $\mathbf{v}$ has a negative (positive) radial component along $I_{1}$ for $H_{+}\left(H_{-}\right)$. A similar reasoning to that discussed above for the horizon limit, means $\mathbf{v}$ winds in the negative (i.e., clockwise) direction along $I_{1}$, when $C$ is circulated in the positive (i.e., counterclockwise) direction, producing another half of a full winding. This means

$$
\Omega_{\theta=\pi}^{\infty}-\Omega_{\theta=0}^{\infty}=-\pi
$$

Total topological charge in the exterior region.-The limits discussed above imply that the topological charge within $\mathcal{I}$, computed from Eq. (6) is $w=-1$, corresponding to a full winding of $\mathbf{v}$ in the negative sense as the contour delimiting $\mathcal{I}$ is circulated in the positive sense. Indeed, Eq. (6) reduces to

$$
w=\frac{1}{2 \pi}\left[\int_{0}^{\pi} d \Omega\right]_{r=\infty}+\frac{1}{2 \pi}\left[\int_{\pi}^{0} d \Omega\right]_{r=r_{H}},
$$

or

$$
w=\frac{1}{2 \pi}\left(\Omega_{\pi}^{\infty}-\Omega_{0}^{\infty}+\Omega_{0}^{\mathcal{H}}-\Omega_{\pi}^{\mathcal{H}}\right)=-1,
$$

where Eqs. (16) and (18) were used in the last equality. This holds for both $H_{ \pm}$and means that there exists at least one standard LR (saddle point of $H_{ \pm}$) for each rotation sense, in the exterior of the $\mathrm{BH}$. Thus, the theorem is proved.

Absence of a horizon.-To understand the key importance of the horizon $\mathcal{H}$, consider the potential $H_{ \pm}$for flat spacetime-see top row of Fig. 3. As expected the essential difference occurs near the left edge of Fig. 3 (top row). The absence of a horizon means $\mathbf{v}$ keeps flowing towards the left in the whole domain, i.e., $v_{r}=-1 /\left(r^{2} \sin \theta\right)<0$, with the sole exception of the axis limit, where it becomes vertical, since $v_{\theta} / v_{r}=\cot \theta \rightarrow \pm \infty$ at $\theta=0, \pi$, respectively. It is the presence of a horizon that introduces the $v_{r}>0$ boundary behavior at the left boundary of the $(r, \theta)$ domain. As our theorem shows, this new boundary behavior must introduce (at least) one LR for each rotation sense.

For flat spacetime, $w=0$ for any contour, and, in particular, one that encloses the full $(r, \theta)$ plane, as it is clear from Fig. 3 (top row). This is true, in fact, as long as the behavior at all boundaries is kept, even for a curved spacetime. Thus, smoothness at the origin and at the axis, together with asymptotic flatness guarantees that the total topological charge will remain zero $w=0$, for any axisymmetric, stationary spacetime, which is smoothly deformable into flat spacetime (and circular). Nonetheless, in such generic smooth horizonless spacetime $\mathbf{v}$ may be locally deformed in the bulk so that LRs emerge. LRs $d o$ not require a horizon. The individual LR charges, however, must add up to zero. In particular, for each standard LR (a saddle point of $H_{ \pm}$, thus with $w=-1$ ) there must be a 
nonstandard LR (maximum or minimum, thus with $w=+1)$. This is the theorem in Ref. [14]. Moreover, if the null energy condition is obeyed, the nonstandard LRs must be stable. Thus, horizonless, asymptotically flat spacetimes with LRs must have a stable LR as long as they are a smooth deformation from flat spacetime, like those originating from an incomplete gravitational collapse [14]. This is illustrated in Fig. 3 (bottom) where $\mathbf{v}$ is exhibited for an ultracompact rotating boson star, a horizonless object in Einstein-Klein-Gordon theory [7,22]. Observe that $w=\{+1,-1,0\}$, respectively, for the contours $\left\{C_{1}, C_{2}, C_{3}\right\}$.

Discussion.-Our theorem puts on a firm ground the hitherto unproved expectation that generic equilibrium BHs must have one standard LR (for each rotation sense), (see also Refs. [23,24]). In addition, it suggests possible ways to circumvent this result. For instance, by dropping: (i) the circularity of the metric. Spacetime circularity holds in vacuum GR BHs but there are reasonable scenarios wherein it can be violated (e.g., toroidal magnetic fields [25]). There is no fundamental reason for circularity to hold for astrophysical BHs; (ii) asymptotic flatness. Changing the asymptotic behavior of the spacetime may change the boundary behavior [Eq. (17)] and hence the whole result. The powerful tool of contour integration and topological LR charge may help us understand more general situations. It seems possible to tackle extremal BHs or nonspherical (e.g., toroidal) horizons in a similar way. Astrophysically one does not expect extremal BHs, which are thus not the focus of this work. Moreover, recall that for extremal Kerr $\mathrm{BHs}$, the Boyer-Lindquist radial coordinate of the corotating LR coincides with that of the horizon. This is a coordinate artifact, but it suggests that the analysis of extremal BHs introduces subtleties.

Finally, some of our assumptions are implied if one focuses on GR with physical matter. For instance, assuming a GR stationary BH spacetime that is asymptotically flat and regularly predictable, with matter satisfying the dominant energy condition, then by Hawking's theorem [26] the cross section of the event horizon has to be topologically spherical $\left(S^{2}\right)$, and the event horizon is a Killing horizon. By further assuming that the spacetime is analytic, nonstatic and with the ergosphere intersecting the horizon, the spacetime is then required to be axially symmetric by Hawking's rigidity theorem.

We thank E. Berti, J. Joudioux, J. Natário, C. Paganini, E. Radu, and M. Rodriguez for discussions. This work is supported by the Center for Research and Development in Mathematics and Applications (CIDMA) through the Portuguese Foundation for Science and Technology (FCT-Fundação para a Ciência e a Tecnologia), references UIDB/04106/2020 and UIDP/04106/2020 and by the projects No. PTDC/FIS-OUT/28407/2017 and No. CERN/
FIS-PAR/0027/2019. This work has further been supported by the European Union's Horizon 2020 research and innovation (RISE) programme H2020-MSCA-RISE-2017 Grant No. FunFiCO-777740. P. C. is supported by the Max Planck Gesellschaft through the Gravitation and Black Hole Theory Independent Research Group. The authors acknowledge networking support by the COST Action CA16104.

[1] B. P. Abbott et al. (Virgo and LIGO Scientific Collaborations), Phys. Rev. Lett. 116, 061102 (2016).

[2] B. P. Abbott et al. (LIGO Scientific and Virgo Collaborations), Phys. Rev. X 9, 031040 (2019).

[3] K. Akiyama et al. (Event Horizon Telescope Collaboration), Astrophys. J. 875, L1 (2019).

[4] K. Akiyama et al. (Event Horizon Telescope Collaboration), Astrophys. J. 875, L5 (2019).

[5] K. Akiyama et al. (Event Horizon Telescope Collaboration), Astrophys. J. 875, L6 (2019).

[6] V. Cardoso, E. Franzin, and P. Pani, Phys. Rev. Lett. 116, 171101 (2016).

[7] P. V. P. Cunha and C. A. R. Herdeiro, Gen. Relativ. Gravit. 50, 42 (2018).

[8] P. V. P. Cunha, C. A. R. Herdeiro, and E. Radu, Phys. Rev. D 96, 024039 (2017).

[9] E. Teo, Gen. Relativ. Gravit. 35, 1909 (2003).

[10] B. Carter, Commun. Math. Phys. 17, 233 (1970).

[11] R. M. Wald, General Relativity (Chicago Univ. Pr., Chicago, USA, 1984).

[12] We do not assume a $\mathbb{Z}_{2}$ north-south spacetime symmetry.

[13] Notice that $-D<0$ is the determinant of the $t-\varphi$ sector of the metric.

[14] P. V. P. Cunha, E. Berti, and C. A. R. Herdeiro, Phys. Rev. Lett. 119, 251102 (2017).

[15] $g_{\varphi \varphi}$ vanishes (only) at the axis. This limit is considered below.

[16] P. V. P. Cunha, J. Grover, C. Herdeiro, E. Radu, H. Runarsson, and A. Wittig, Phys. Rev. D 94, 104023 (2016).

[17] See Supplemental Material at http://link.aps.org/ supplemental/10.1103/PhysRevLett.124.181101 for some additional technical details.

[18] S. Hod, Phys. Lett. B 776, 1 (2018).

[19] One can also regard $w$ as the Brouwer degree of the map defined by the angle $\Omega$, namely $\Omega: S^{1} \rightarrow S^{1}$, which maps each point of $C$ to a point in an auxiliary circle. This was the perspective taken in Ref. [14].

[20] J. Carot, Classical Quantum Gravity 17, 2675 (2000).

[21] A. J. M. Medved, D. Martin, and M. Visser, Phys. Rev. D 70, 024009 (2004).

[22] R. Ruffini and S. Bonazzola, Phys. Rev. 187, 1767 (1969).

[23] S. Hod, Phys. Lett. B 727, 345 (2013).

[24] C. Paganini, The role of trapping in black hole spacetimes, Ph. D. thesis, Universität Potsdam, 2018.

[25] E. Gourgoulhon and S. Bonazzola, Phys. Rev. D 48, 2635 (1993).

[26] S. W. Hawking, Commun. Math. Phys. 25, 152 (1972). 